\title{
Time-dependent simulation of free-electron laser amplifiers and oscillators
}

\author{
Henry P. Freund \\ Science Applications International Corporation, McLean, Virginia 22102 USA
}

(Received 19 September 2005; published 17 November 2005)

\begin{abstract}
Time-dependent free-electron laser simulations use a variety of techniques. Particle-in-cell codes have been used to simulate free-electron masers; however, this is not feasible at short wavelengths. Most simulations use a slowly varying envelope approximation in both in $z$ and $t$, where the particles and fields are advanced in $z$ using the same process as in steady-state simulations and then the derivative describing slippage is applied. We describe the inclusion of this technique in the non-wiggler-averaged code MEDUSA, which is then applied to study temporal behavior in amplifiers and oscillators.
\end{abstract}

DOI: 10.1103/PhysRevSTAB.8.110701

PACS numbers: 41.60.Cr

\section{INTRODUCTION}

Time-dependent free-electron laser (FEL) simulations use a variety of techniques. Particle-in-cell codes have been used to simulate free-electron masers [1,2]; however, this is not feasible at short wavelengths. Most simulations use a slowly varying envelope approximation (SVEA). One such technique assumes that the envelope varies only in $z$ combined with a field representation as an ensemble of discrete harmonics. This has been shown to be equivalent to a time-dependent simulation [3]; however, it is often computationally prohibitive. A second technique uses an SVEA in both in $z$ and $t$ [4], and the particles and fields are advanced in $z$ using the same process as in steady-state simulations and then the time derivative describing slippage is applied. This is used in wiggleraveraged codes such as PERSEO [5] in 1D and GINGER [6] and GENESIS [7] in 3D. We describe the inclusion of this technique in the non-wiggler-averaged code MEDUSA [8], which is then applied to amplifiers and oscillators.

\section{THE NUMERICAL FORMULATION}

The formulation follows closely on that for the steadystate formulation [8]. The electromagnetic field is represented as a superposition of Gauss-Hermite modes

$$
\begin{aligned}
\delta \mathbf{A}(\mathbf{x}, t)= & \hat{\mathbf{e}}_{x} \sum_{l, n, h} e_{l, n, h}(x, y)\left[\delta A_{l, n, h}^{(1)} \cos \varphi_{h}\right. \\
& \left.+\delta A_{l, n, h}^{(2)} \sin \varphi_{h}\right],
\end{aligned}
$$

where " $l$ " and " $n$ " are transverse mode numbers, " $h$ " is the harmonic number, $e_{l, n, h}=\exp \left(-r^{2} / w_{h}{ }^{2}\right) H_{l}\left(\sqrt{2 x / w_{h}}\right) \times$ $H_{n}\left(\sqrt{2 y / w_{h}}\right), H_{l}$ is the Hermite polynomial of order $l$, and $w_{h}$ is the spot size, $\varphi_{h}=h\left(k_{0} z-w_{0} t\right)+\alpha_{h} r^{2} / w_{h}^{2}\left(k_{0}=\right.$ $\left.\omega_{0} / c\right)$. We assume that $\delta A_{l, n, h}^{(1,2)}, w_{h}$, and $\alpha_{h}$, vary slowly in $z$ and $t$. The dynamical equations are

$$
\left(\frac{d}{d z}+\frac{w_{h}{ }^{\prime}}{w_{h}}\right)\left(\begin{array}{c}
\delta a_{l, n, h}^{(1)} \\
\delta a_{l, n, h}^{(2)}
\end{array}\right)+K_{l, n, h}\left(\begin{array}{c}
\delta a_{l, n, h}^{(2)} \\
-\delta a_{l, n, h}^{(1)}
\end{array}\right)=\left(\begin{array}{c}
s_{l, n, h}^{(1)} \\
s_{l, n, h}^{(2)}
\end{array}\right)
$$

where $\delta a_{l, n, h}^{(1,2)}=e \delta A_{l, n, h}^{(1,2)} / m_{e} c^{2}, d / d z=\partial / \partial z+c^{-1} \partial / \partial t$, the "prime" superscript denotes the total $z$ derivative,

$$
K_{l, n, h}=(l+n+1)\left(\alpha_{h} \frac{w_{h}{ }^{\prime}}{w_{h}}-\frac{\alpha_{h}{ }^{\prime}}{2}-\frac{1+\alpha_{h}^{2}}{h k_{0} w_{h}^{2}}\right),
$$

$$
\left(\begin{array}{c}
s_{l, n, h}^{(1)} \\
s_{l, n, h}^{(2)}
\end{array}\right)=\frac{2 \omega_{b}^{2}}{h \omega_{0} c} \frac{F_{l, n}}{w_{h}^{2}}\left\langle\frac{v_{x}}{\left|v_{z}\right|} e_{l, n, h}\left(\begin{array}{c}
\cos \varphi_{h} \\
-\sin \varphi_{h}
\end{array}\right)\right\rangle
$$

where $\omega_{b}(z, t)^{2}=4 \pi e^{2} n_{b}(z, t) / m_{e}$ for a beam density $n_{b}$, and $F_{l, n}=\left[2^{l+n} l ! n !\right]^{-1}$. Note that the beam density varies to account for the temporal profile of the pulse. For a beam with a Gaussian energy and phase space distributions, the source terms are

$$
\langle(\cdots)\rangle \equiv \int_{0}^{2 \pi} \frac{d \psi_{0}}{2 \pi} \int_{0}^{\infty} d \gamma_{0} \frac{\exp \left[-\left(\gamma-\bar{\gamma}_{0}\right)^{2} / 2 \Delta \gamma^{2}\right]}{\sqrt{\pi / 2} \Delta \gamma\left[1+\operatorname{erf}\left(\bar{\gamma}_{0} / \sqrt{2} \Delta \gamma\right)\right]} \iiint \int d x_{0} d y_{0} d p_{x 0} d p_{y 0} \frac{\exp \left(-r_{0}^{2} / 2 \sigma_{r}^{2}-p_{\perp 0}^{2} / 2 \sigma_{p}^{2}\right)}{(2 \pi)^{2} \sigma_{r}^{2} \sigma_{p}^{2}}(\cdots)
$$

and we assume, for simplicity, that only the current varies over the course of the pulse. The spot size and radius of curvature for each harmonic component are given by

$$
w_{h}^{\prime}=\frac{2 \alpha_{h}}{h k_{0} w_{h}}-w_{h} Y_{h}
$$

$$
\frac{\alpha_{h}^{\prime}}{2}=\frac{1+\alpha_{h}^{2}}{h k_{0} w_{h}^{2}}-\left(X_{h}+\alpha_{h} Y_{h}\right)
$$




$$
\begin{gathered}
X_{h}=2 \frac{\left(s_{2,0, h}^{(1)}+s_{0,2, h}^{(1)}\right) \delta a_{0,0, h}^{(2)}-\left(s_{2,0, h}^{(2)}+s_{0,2, h}^{(2)}\right) \delta a_{0,0, h}^{(1)}}{\delta a_{0,0, h}{ }^{2}}, \\
Y_{h}=-2 \frac{\left(s_{2,0, h}^{(1)}+s_{0,2, h}^{(1)}\right) \delta a_{0,0, h}^{(1)}+\left(s_{2,0, h}^{(2)}+s_{0,2, h}^{(2)}\right) \delta a_{0,0, h}^{(2)}}{\delta a_{0,0, h}{ }^{2}},
\end{gathered}
$$

and $\delta a_{0,0, h}^{2}=\delta a_{0,0, h}^{(1)}+\delta a_{0,0, h}^{(2)}$. These equations constitute the source-dependent expansion [9], which is a selfconsistent adaptive eigenmode representation that tracks the optical guiding of the mode based upon the interaction with the electron beam.

Some discussion of the numerical procedures is appropriate before proceeding with a discussion of simulation results for amplifiers and oscillators. These field equations are integrated simultaneously with the complete Lorentz force equations for an ensemble of electrons including the optical mode, the wiggler(s), and any additional focusing fields or magnetic dispersive elements (such as a chicane). Because MEDUSA uses a non-wiggler-averaged formalism, the step size in $z$ must be small enough to resolve the wiggler motion and the step size for the simulations discussed in the succeeding sections is $1 / 30$ th of a wiggler period.

The electron bunch is represented by a sequence of "slices" each of which corresponds to a "beamlet" one wavelength long. The radiation field is also represented as a superposition of slices corresponding to the slices of the electron bunch. The initial conditions for both the electron bunch and the radiation pulse are chosen to represent parabolic pulse shapes. This is illustrated in Fig. 1 where we show the electron bunch at the top and the radiation pulse below normalized to the peak current, $I_{\text {peak }}$, and the peak input power, $P_{\text {peak }}$, respectively. The separation between the slices, $\Delta t$, may be varied but, in practice, we find that the slice spacing must be about one every three wavelengths. MEDUSA allows for an offset in the centers of the electron and radiation pulses (shown as $t_{\text {offset }}$ in the figure) in order to describe (1) injection of seed power in an amplifier that is not precisely synchronized with the electron bunches (although this is not considered in this paper), and (2) for cavity tuning in an oscillator where the roundtrip time for the radiation may be chosen to give arbitrary synchronism between the radiation and the electrons from pass to pass. Because the radiation slips ahead of the electrons allowance must be made in the simulation for radiation slices that have no corresponding electron slice, and these are referred to as "blank" slices in the figure. Note that the blank slices initially are at zero power and grow only due to slippage or specific cavity tuning, and that blank slices are only needed at the trailing edge of the radiation pulse for oscillator simulations. In this work, we only consider variations in the beam current and radiation

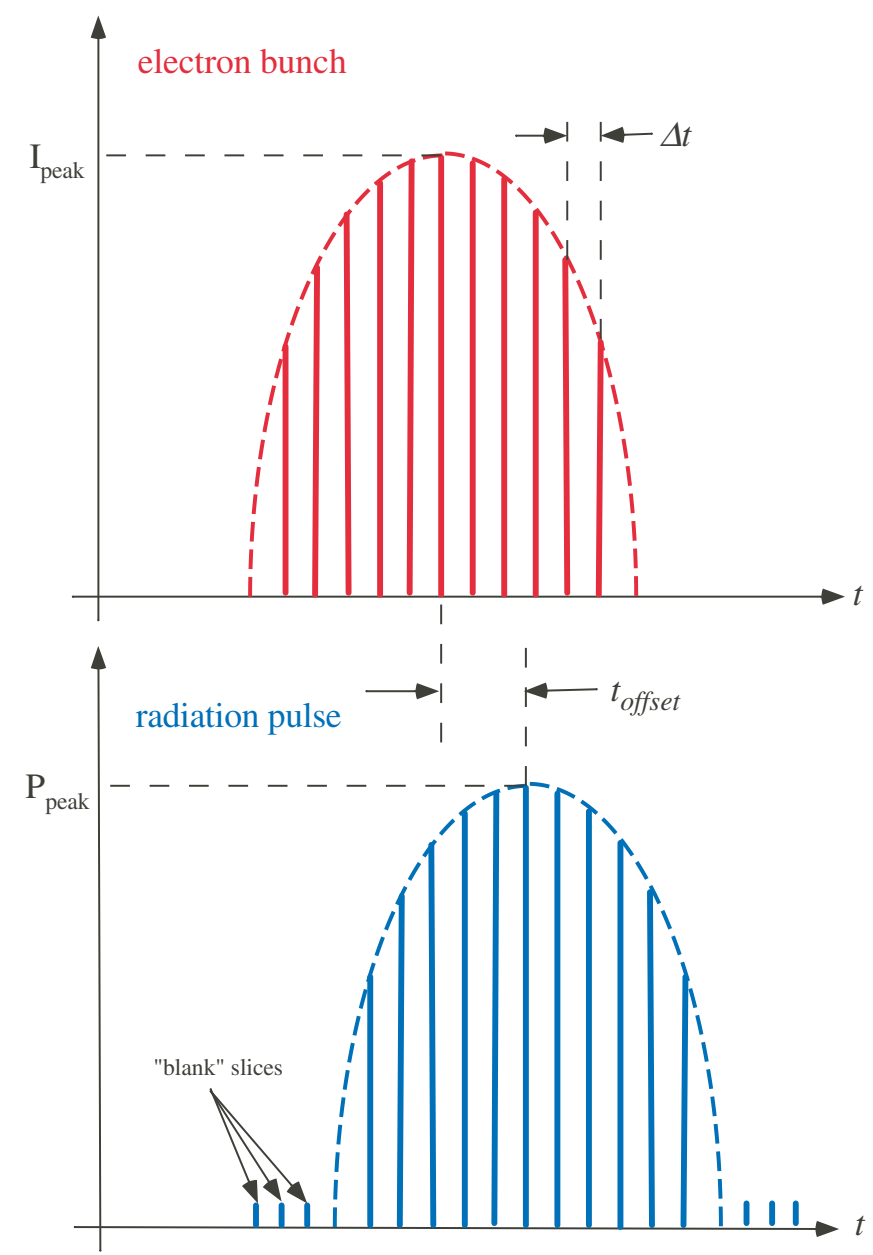

FIG. 1. (Color) Schematic illustration of the initial electron bunch and radiation pulse.

power from slice-to-slice for simplicity. More generally, however, variation in beam energy, emittance, and energy spread must also be included for the electron bunch as well as variations in spot size and the wave front curvature for the radiation pulse.

The numerical procedure follows that used in Refs. [47]. Specifically, each slice is advanced from $z \rightarrow z+\Delta z$ using the source terms (i.e., the source current due to the electron beam corresponding to that slice) by means of a 4 th order Runge-Kutta algorithm. Thus far, the procedure follows that employed in steady-state simulations. The time dependence (i.e., slippage) is imposed as an additional operation using the forward time derivative as an additional source term that couples the $i$ th radiation slice to the $(i+1)$ th slice. In practice, it may be imposed on every spatial step, only after some spatial interval. In this way information is allowed to propagate only in the forward direction. In practice, this means that the radiation slices communicate via $\delta \mathbf{A}(t)=\delta \mathbf{A}(t)-\left(\lambda_{w} / \delta z\right)(\lambda / c \Delta t) \times$ $[\delta \mathbf{A}(t)-\delta \mathbf{A}(t-\Delta t)]$, where $\delta z(\geq \Delta z)$ represents the spatial separation between the applications of this slippage operation. Therefore, if the slippage operation is applied 
once every wiggler period $\left(\delta z=\lambda_{w}\right)$ and the radiation slices are separated by a single wavelength $(c \Delta t=\lambda)$, then we obtain $\delta \mathbf{A}(t)=\delta \mathbf{A}(t-\Delta t)$ and the radiation slices advance one wavelength per wiggler period, as expected.

In practice the interval between applications of the slippage operation can be varied; however, in all the cases discussed in this paper, the slippage operation is applied on every spatial step $(\delta z=\Delta z)$. This ensures that the wave-particle interaction is treated self-consistently in view of the fact that MEDUSA does not employ a wiggleraveraged orbit approximation.

\section{AMPLIFIER MODELING}

In simulating amplifiers, the initial radiation pulse is chosen to correspond to the electron pulse for a given peak seed power. As the electrons and radiation propagate through the wiggler, the radiation slips ahead of the electrons (one wavelength per wiggler period at resonance); hence, the simulation must include additional slices of the radiation pulse ahead of the electrons but which start at zero power and have no corresponding electron slice.

As an example showing slippage in amplifiers, we use a $76 \mathrm{MeV} / 400 \mathrm{~A}$ electron beam with an emittance of $15 \mathrm{~mm}$-mrad, an energy spread of $0.1 \%$, and a pulse time of $1 \mathrm{psec}$. The wiggler amplitude is $7.5 \mathrm{kG}$ with a period of $2.18 \mathrm{~cm}$, which yields a resonance at a wavelength of $1.06 \mu \mathrm{m}$. A seed power of $1 \mathrm{~W}$ is assumed. The growth of the pulse energy through the wiggler is shown in Fig. 2. Exponential growth is evident and saturation is found after about $7.5 \mathrm{~m}$ with a $25 \mu \mathrm{J}$ pulse energy corresponding to a peak power of $74 \mathrm{MW}$.

Radiation slippage through the wiggler results in a pulse with both slips ahead of the electrons and distorts in shape from the original parabolic profile. This is illustrated in Fig. 3 where we plot the normalized power in the pulse at various points in the wiggler. The initial pulse is parabolic with a 1 psec duration, and the center of the electron bunch

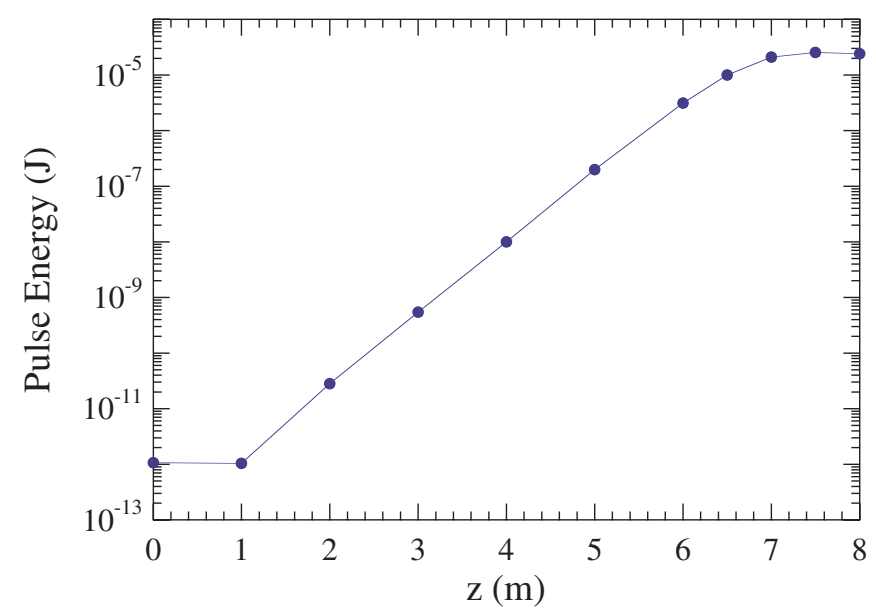

FIG. 2. (Color) Growth of the pulse energy through the wiggler.

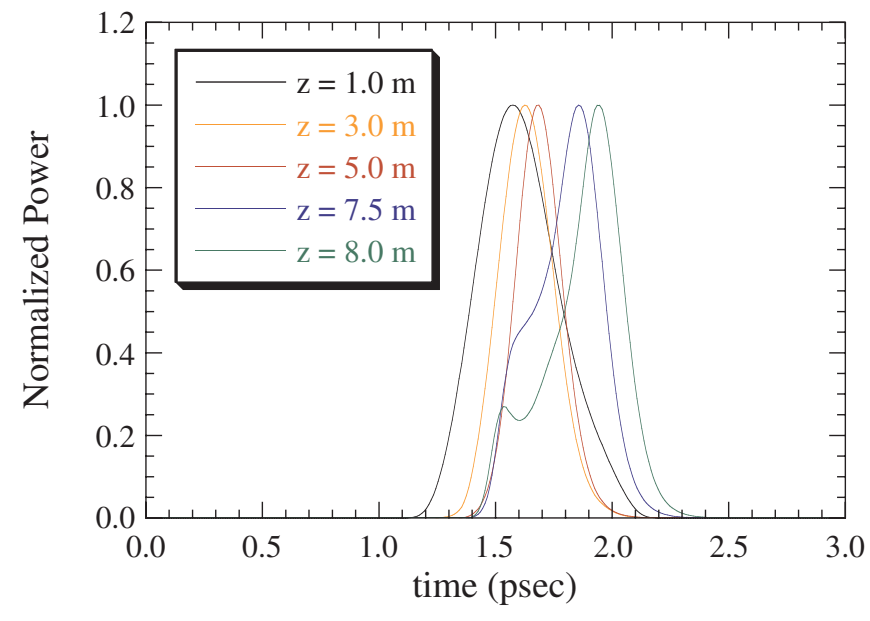

FIG. 3. (Color) Evolution of radiation pulse shapes versus time.

is at $1.5 \mathrm{psec}$ in the figure. It is clear that as the interaction proceeds, the radiation slips significantly ahead of the electrons and is distorted in shape and narrowed in duration.

Since the slippage time is comparable to the pulse duration, we expect that slippage will significantly reduce the power and pulse energy relative to that from a steadystate simulation. For comparison, we show the results of a steady-state simulation in Fig. 4 where we find a much higher peak power of $113 \mathrm{MW}$. If we assume that the pulse is parabolic and does not distort over the course of the interaction, then the pulse energy is given by $E_{\text {pulse }}=$ $(2 / 3) P \tau_{\text {pulse }}$. This implies that the pulse energy at saturation would be about $75 \mu \mathrm{J}$. This dramatically illustrates the reductions in performance due to slippage when the slippage time is comparable to the pulse duration.

\section{OSCILLATOR MODELING}

The procedure for modeling oscillators in MEDUSA is illustrated schematically in Fig. 5. In contrast to amplifiers

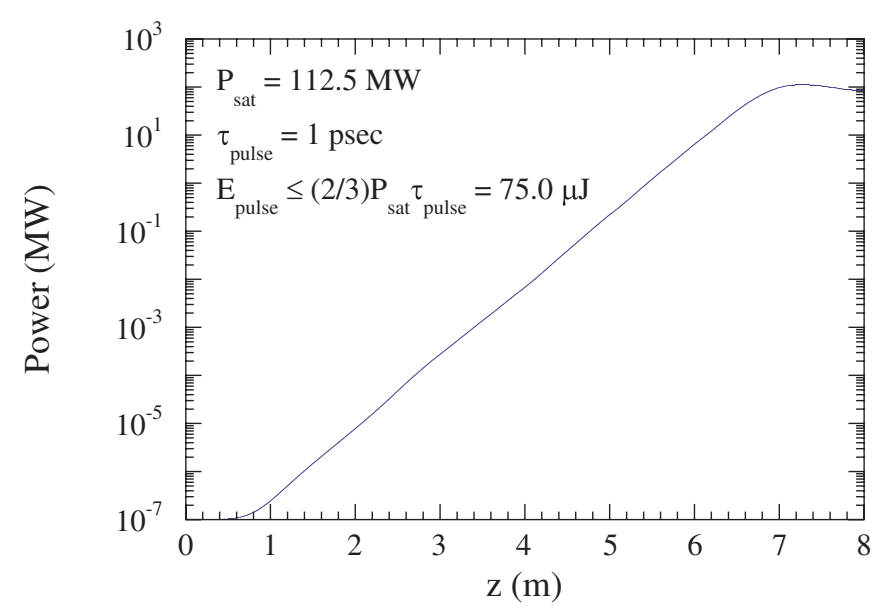

FIG. 4. (Color) Power growth in a steady-state simulation. 


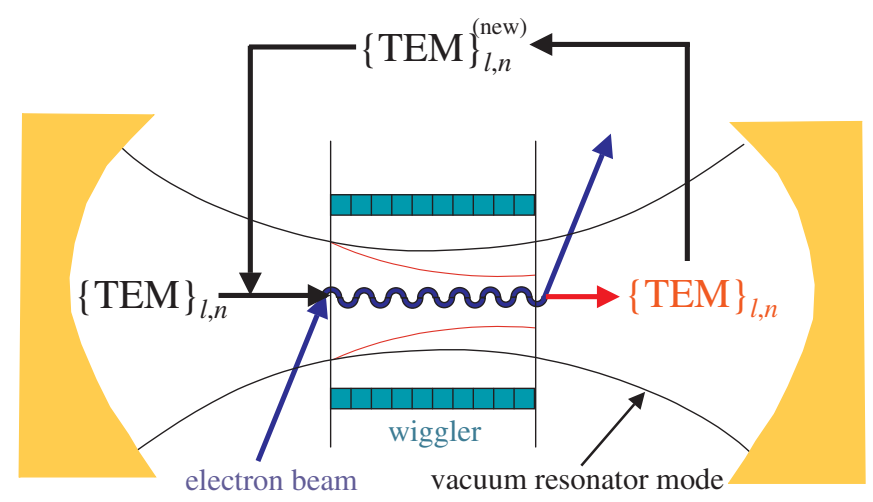

FIG. 5. (Color) Schematic of oscillator feedback.

where the optical mode is guided by the electron beam, the mode in oscillators is largely governed by the resonator design although the degree of optical guiding increases as the gain per pass increases. The wiggler is located in the center of the resonator, and the electron beam is injected into the wiggler/resonator and amplifies what is initially (i.e., at the entrance to the wiggler) an ensemble of vacuum resonator modes $\left[\{\mathrm{TEM}\}_{l, n}\right]$. The mode is both amplified and guided in the wiggler; hence, it is focused to a smaller spot at the wiggler exit than would be found in the vacuum resonator. This "dressed" ensemble of resonator modes $\left[\{\mathrm{TEM}\}_{l, n}\right.$ (indicated in red in Fig. 5) $]$ is then decomposed into a new ensemble of vacuum resonator modes $\left[\{\operatorname{TEM}\}_{l, n}^{(\text {new })}\right]$, which is "propagated" back to the wiggler entrance where it interacts with another electron bunch. This process is repeated an arbitrary number of times until the system reaches a steady state.

For a confocal resonator, the mode waist of the vacuum resonator, $w_{0}$, is located at some point within the wiggler denoted by $z_{0}$ so that the spot size and curvature vary as $w(z)=w_{0}\left[1+\left(z-z_{0}\right)^{2} / z_{R}{ }^{2}\right]^{1 / 2}$ and $\alpha(z)=\left(z-z_{0}\right) / z_{R}$, where the Rayleigh range in vacuo is given by $z_{R}=$ $\pi w_{0}^{2} / \lambda$. In the absence of any interaction with the electron beam, the (vacuum) resonator mode is characterized by a spot size of $w_{v}=w_{0}\left[1+L_{w}{ }^{2} / 4 z_{R}{ }^{2}\right]^{1 / 2}$ and a curvature of $\alpha_{v}=L_{w} / 2 z_{R}$ at the exit of a wiggler of length $L_{w}$, where we have assumed that the centers of the wiggler and resonator coincide. We use the subscript " $v$ " to denote the vacuum modal properties. Of course, the dressed mode will be characterized by a different spot size and curvature, and we must decompose the dressed mode into a new ensemble of vacuum modes.

This decomposition can be accomplished in a straightforward manner. If we denote the new vacuum modes by $\delta \bar{A}_{l, n}^{(1,2)}$ where we have dropped the subscript over the harmonic number for convenience, then we find that

$$
\left(\begin{array}{l}
\delta \bar{A}_{l, n}^{(1)} \\
\delta \bar{A}_{l, n}^{(2)}
\end{array}\right)=\frac{1}{2^{l+n} \pi l ! n !} \sum_{l^{\prime}, n^{\prime}}\left(\begin{array}{cc}
C_{l^{\prime}, n^{\prime}}^{(l, n)} & S_{l^{\prime}, n^{\prime}}^{(l, n)} \\
-S_{l^{\prime}, n^{\prime}}^{(l, n)} & C_{l^{\prime}, n^{\prime}}^{(l, n)}
\end{array}\right)\left(\begin{array}{l}
\delta A_{l^{\prime}, n^{\prime}}^{(1)} \\
\delta A_{l^{\prime}, n^{\prime}}^{(2)}
\end{array}\right),
$$

where the matrix coefficients are

$$
\begin{aligned}
& C_{l^{\prime}, n^{\prime}}^{(l, n)}=I_{l, l^{\prime}}^{(c)} I_{n, n^{\prime}}^{(c)}-I_{l, l^{\prime}}^{(s)} I_{n, n^{\prime}}^{(s)}, \\
& S_{l^{\prime}, n^{\prime}}^{(l, n)}=I_{l, l^{\prime}}^{(s)} I_{n, n^{\prime}}^{(c)}+I_{l, l^{\prime}}^{(c)} I_{n, n^{\prime}}^{(s)},
\end{aligned}
$$

and

$$
\begin{aligned}
I_{l, l^{\prime}}^{(c)}= & \int_{-\infty}^{\infty} d x e^{-x^{2} / w^{2}-x^{2} / w_{v}^{2}} H_{l}\left(\frac{\sqrt{2} x}{w_{v}}\right) H_{l^{\prime}}\left(\frac{\sqrt{2} x}{w}\right) \\
& \times \cos \left[\left(\frac{\alpha_{v}}{w_{v}^{2}}-\frac{\alpha}{w^{2}}\right) x^{2}\right], \\
I_{l, l^{\prime}}^{(s)}= & \int_{-\infty}^{\infty} d x e^{-x^{2} / w^{2}-x^{2} / w_{v}^{2}} H_{l}\left(\frac{\sqrt{2} x}{w_{v}}\right) H_{l^{\prime}}\left(\frac{\sqrt{2} x}{w}\right) \\
& \times \sin \left[\left(\frac{\alpha_{v}}{w_{v}^{2}}-\frac{\alpha}{w^{2}}\right) x^{2}\right] .
\end{aligned}
$$

These integrals can be performed very efficiently by numerical methods on each pass of the optical mode through the wiggler/resonator. It should also be noted that this technique can be used both in the steady-state regime and when slippage is included.

In simulating slippage in oscillators, the initial condition for the first pass around the resonator is the same as for the treatment of amplifiers, but subsequent passes must account for cavity detuning. Depending on the cavity length, the radiation pulse may arrive ahead or behind the electron bunch. Therefore, it is necessary to include blank radiation slices both ahead and behind the electron bunch and to shift the overlap of the radiation and electron slices on each pass based on the electron bunch repetition rate and the radiation round trip time in the resonator.

The example we consider employs a $47.15 \mathrm{MeV} / 60 \mathrm{~A}$ (peak current) electron beam with an emittance of $7 \mathrm{~mm}$ $\mathrm{mrad}$ in the wiggler plane and $8 \mathrm{~mm}$-mrad in the plane normal to the wiggle plane. For computational simplicity, we also assume that the energy spread vanishes. The pulse duration is assumed to be $1 \mathrm{psec}$ and the pulse repetition rate is $37 \mathrm{MHz}$, which corresponds to a duty factor of $3.7 \times$ $10^{-5}$. We assume that the wiggler amplitude is $5.5 \mathrm{kG}$, the period is $2.7 \mathrm{~cm}$, and the length is 41 periods. This yields a resonance at a wavelength of $3.11 \mu \mathrm{m}$. The waist size in the resonator is taken to be $628 \mu \mathrm{m}$, and we assume that $10 \%$ of the power is out coupled. The optimal cavity tuning is dependent upon the transit time through the resonator and the repetition rate of the electron bunches. The optimal cavity length is found to be $8.102485 \mathrm{~m}$ for which we find that the peak pulse energy in the resonator is $67 \mu \mathrm{J}$. In view of the duty factor and resonator out coupling, this yields an average output power of about $250 \mathrm{~W}$. The evolution of the pulse energy versus pass is shown in Fig. 6 for a variety of different cavity lengths in the neighborhood of the optimum cavity length, and shows a fairly rapid decline in 


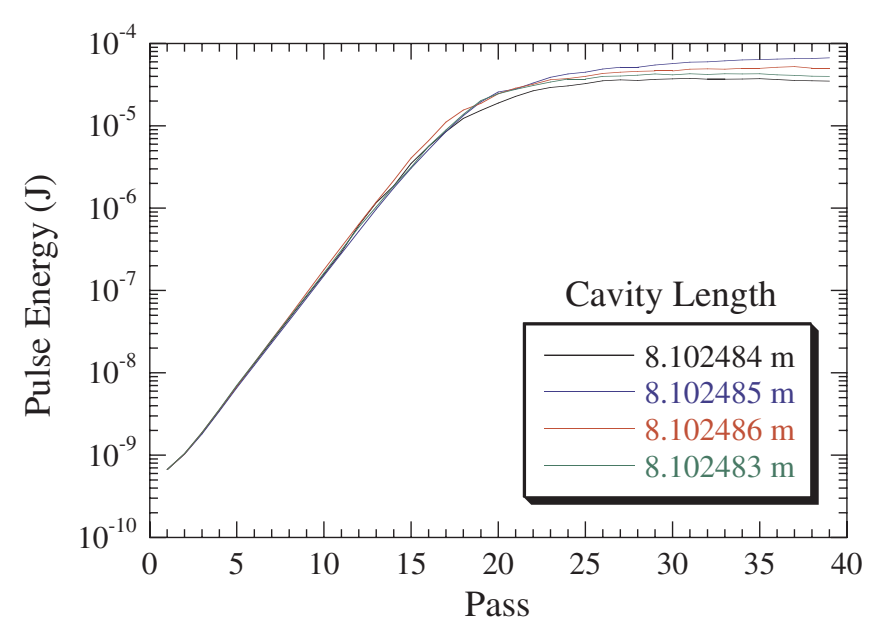

FIG. 6. (Color) Growth of the pulse energy versus pass.

pulse energy as the cavity length shifts away from the optimum value.

Accounting for this slippage as well as the cavity tuning, the distortion of the radiation pulse corresponding to the optimal cavity length after 40 passes is given in Fig. 7, and shows a sharp trailing edge with a more gradual decline in the leading edge. We also observe that, due to the cavity tuning, the total pulse width has broadened although the FWHM width remains about 1 psec.

The slippage time through the wiggler for this example is about $0.4 \mathrm{psec}$. This is comparable to pulse duration, and we would expect that slippage has a negative effect on the oscillator power. This is illustrated in a steady-state simulation shown in Fig. 8, where we note that in comparison, the steady-state simulation for these oscillator parameters yields a peak power of $170 \mathrm{MW}$. Assuming a parabolic

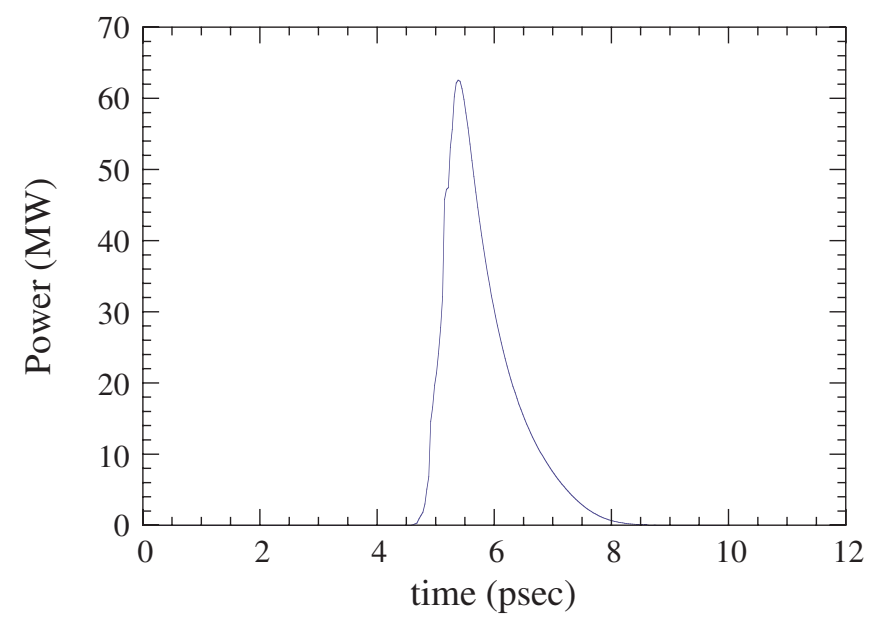

FIG. 7. (Color) Pulse shape after 40 passes through the wiggler for a cavity length of $8.102485 \mathrm{~m}$.

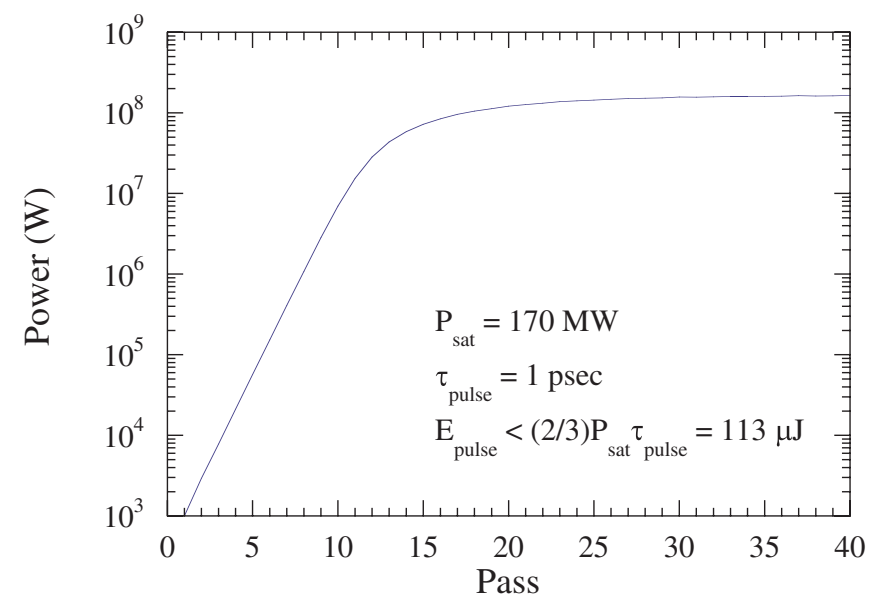

FIG. 8. (Color) Steady-state oscillator simulation.

pulse shape that is undistorted by the interaction, we note that this corresponds to a peak pulse energy of about $113 \mu \mathrm{J}$ and an average output power of about $629 \mathrm{~W}$.

\section{SUMMARY}

The associated effects of time dependence and slippage have been incorporated into the non-wiggler-averaged MEDUSA simulation code, and the results applied to both amplifier and oscillator configurations. As expected, when the slippage time is comparable to the duration of the electron pulses, slippage is seen to have a significant negative impact on the FEL interaction.

\section{ACKNOWLEDGMENTS}

This work was supported by the Joint Technology Office and the Office of Naval Research.

[1] T. J. T. Kwan, J. M. Dawson, and A. T. Lin., Phys. Fluids 20, 581 (1977).

[2] A. T. Lin, C. C. Lin, T. Taguchi, and W. W. Cheng, Phys. Fluids 26, 3 (1983).

[3] N. Piovella, Phys. Plasmas 6, 3358 (1999).

[4] R. Bonifacio, B. W. J. McNeil, and P. Pierini, Phys. Rev. A 40, 4467 (1989).

[5] L. Giannessi, PERSEO reference available at www.perseo.enea.it

[6] W. Fawley, LBID-2141, CBP Tech Note-104, UC-414, 1995.

[7] S. Reiche, Nucl. Instrum. Methods Phys. Res., Sect. A 429, 243 (1999).

[8] H.P. Freund, S. G. Biedron, and S. V. Milton., IEEE J. Quantum Electron. 36, 275 (2000).

[9] P. A. Sprangle, A. Ting, and C. M. Tang, Phys. Rev. A 36, 2773 (1987). 